\title{
EFECTO EN EL ÍNDICE DE DISCAPACIDAD EN PACIENTES ADULTOS CON VÉRTIGO PAROXÍSTICO POSICIONAL BENIGNO MEDIANTE LA REHABILITACIÓN VESTIBULAR CON MOVIMIENTO HUMANO
}

\author{
Sofía Chaverri Flores ${ }^{1}$, Julián Chaverri Polini ${ }^{2}$ y Andrea Mora Campos $^{3}$ \\ ${ }^{1}$ Clínica Aguilar Bonilla, sofchaflo@hotmail.com \\ ${ }^{2}$ Hospital México, juliancp@racsa.co.cr \\ ${ }^{3}$ Universidad Nacional, andremor31@gmail.com \\ Costa Rica \\ 2007
}

\section{RESUMEN}

\begin{abstract}
Propósito: determinar el efecto en el índice de discapacidad en pacientes adultos con vértigo paroxístico posicional benigno mediante la $R V$ con el movimiento humano. Sujetos: seis sujetos, con una edad promedio de $49.5 \pm 14.22$ años, que se han diagnosticado previamente, por medio de un especialista en otorrinolaringología, con vértigo paroxístico posicional benigno. Instrumentos: se utilizó el Dizziness Handicap Inventory, cuestionario para determinar el impacto en la calidad de vida en pacientes con dicha patología (Ceballos y Vargas, 2004). Procedimiento: se les sometió a una terapia vestibular durante cuatro semanas con ejercicios de habituación y equilibrio, en una modalidad semipresencial. Se realizaron dos mediciones, antes y después de terapia vestibular, y se valoró si hubo alguna mejoría en las dimensiones física, funcional y emocional. Análisis estadístico: se aplicó estadística descriptiva y una "t-Student" de medidas repetidas para analizar los resultados obtenidos. Resultados: hubo diferencias estadísticamente significativas en la dimensión física entre el pretest $(19.33 \pm 4.67$ puntos $)$ y el postest $(13 \pm 7.24$ puntos $)(t=2.65 ; p<0.05)$. Por otro lado, no hubo diferencias significativas en las dimensiones funcional $(t=2.44 ; p>0.05)$, emocional $(t=2.37 ; p>0.05) y$ general ( $t=2.55 ; p>0.05)$. Conclusión: la terapia vestibular con movimiento humano semipresencial mejoró el índice de discapacidad por vértigo (dimensión física) de sujetos con VPPB.
\end{abstract}

EFFECT ON THE DISABILITY INDEX OF ADULT PATIENTS WITH BENIGN PAROXYSMAL POSITIONAL VERTIGO USING VESTIBULAR REHABILITATION AND HUMAN MOVEMENT

ABSTRACT

\begin{abstract}
Objective: determine the effect on the disability index of adult patients with benign paroxysmal positional vertigo $(B P P V)$ using vestibular rehabilitation therapy (VRT) and human movement. Subjects: six subjects with an average age of $49.5 \pm 14.22$ years who have been diagnosed with benign paroxysmal positional vertigo by an otolaryngologist. Instruments: the Dizziness Handicap Inventory and a questionnaire to determine impact on the quality of life of patients with this pathology (Ceballos and Vargas, 2004). Procedure: subjects underwent vestibular therapy for four weeks together with habituation and balance exercises in a semi-supervised manner. Two measurements were performed, one before and one after the vestibular therapy and researchers determined if there was any improvement in the physical, functional, and emotional dimensions. Statistical analysis: descriptive statistics and Student's t-test of repeated measures were applied to analyze results obtained. Results: significant statistical differences were found in the physical dimension between the pre-test (19.33 \pm 4.67 points $)$ and post-test $(13 \pm 7.24$ points $)(t=2.65 ; p<0.05)$. In contrast, no significant statistical differences were found in the functional $(t=2.44 ; p>0.05)$, emotional $(t=2.37 ; p>0.05)$ or general dimensions $(t=2.55 ; p>0.05)$. Conclusion: vestibular therapy with a semi-supervised human movement program improved the index of disability due to vertigo (physical dimension) in BPPV subjects.
\end{abstract}




\section{INTRODUCCIÓN}

La palabra equilibrio proviene del latín: libra que significa balance. La función de este se puede definir como la actividad que gobierna o dirige las relaciones del animal con el mundo físico permitiéndole asegurar todos sus movimientos en una forma eficaz (Roman y Thomassin, 2000). La función del equilibrio integra la noción de una plurimodalidad sensorial en donde interviene: el sistema vestibular quien informa sobre la aceleración tanto lineal como angular y la posición de la cabeza, y su eje de inclinación en relación al gravitacional; la visión, que ayuda a ubicar y a dar información sobre lo que se mueve alrededor de la persona; la propiocepción es quien ayuda a informar sobre la disposición de los diferentes segmentos del cuerpo y de la relación que guardan entre sí (Hassid, Hennaux y Van Nechel, 2004).

Bartual y colaboradores (1998), describen que sólo algunas de las formas más primitivas de la vida, como las bacterias, algas verduscas y muchos organismos unicelulares, como los flagelados, se han adaptado al medio ambiente sin desarrollar receptores especializados para detectar, al menos, la fuerza de la gravedad pero en algunos animales como los peces y pájaros, la pérdida del laberinto, es incompatible con la vida. A partir de los vertebrados superiores hasta llegar al ser humano, existen otros factores que intervienen en el equilibrio como son la visión y la sensibilidad profunda. El ser humano vive en un mundo de cuatro dimensiones que están íntimamente relacionadas. Las tres primeras corresponden al espacio, y la cuarta al tiempo.

La persona sana debe estar permanentemente orientada en el espacio y en el tiempo para mantenerse en equilibrio y poder desarrollar su actividad (Bartual y colaboradores, 1998). Pero no siempre es de esta forma, y una de las afecciones que puede aquejar es el síndrome vertiginoso, el cual según Tran Ba Huy y de Waele (1996), puede ser de origen periférico o sea por patología del oído, del nervio cocleovestibular o de origen central por patología de la vía vestibular o lesión a nivel superior. Entre las patologías más frecuentes de origen periférico, existen el Vértigo Paroxístico Posicional Benigno (VPPB), la neuronitis vestibular y el Síndrome de Meniere; entre las de origen central una de las más frecuentes es el vértigo del adulto mayor.

El vértigo es un síntoma no un diagnóstico y manifiesta una alteración de la orientación en el espacio. Se trata de sensaciones de movimiento de carácter giratorio, de desplazamientos de la base de sustentación, inclinación del suelo, precipitación en el vacío y sensación de caída al adoptar determinadas posturas. Se diferencia del desequilibrio, ya que este consiste en sensaciones de balanceo, de inseguridad e inestabilidad (Toupet e Imbaud-Genieys, 2002). Según Oghalai, Manolidis, Barth, Stewart y Jenkins (2000) y Whitney, Hudak y Marchetti (1999), las personas con este padecimiento se ven afectadas negativamente en las actividades diarias, por lo tanto en la calidad de vida.

Según Bouccara, Sémont y Sterkers (2003), los conocimientos básicos que existen para tratar la compensación vestibular, han permitido el desarrollo de la reeducación de esta. Una vez que se realiza una evaluación otoneurológica que permita un diagnóstico etiológico del vértigo, se puede dar un tratamiento y escoger las técnicas de reeducación que se adapten más a la patología que se quiere tratar. La mayoría de las enfermedades que requieren de reeducación vestibular, necesitarán además un manejo multidisciplinario, sobretodo si se trata de un paciente de la tercera edad (Norré y Beckers, 1988), además está indicada en pacientes que presentan vértigo o desequilibrio, ya sea de origen central o periférico, y conformada por ejercicios vestibulares, que además son una interesante alternativa para tratar específicamente VPPB (Hassid, 2002).

Los ejercicios de habituación constituyen la primera técnica de rehabilitación vestibular (RV) formalmente descrita, se establecen en la década de los años 40 con los trabajos de Cawthorne y 
Cooksey en 1944 (Bouccara, Sémont y Sterkers, 2003). Toledo, Cortés, Pane y Trujillo (2000), recomiendan que el tratamiento basado en ejercicios de RV es efectivo en el $90 \%$ de los casos, pero debe ser realizado por lo menos tres a cuatro veces por semana. También mencionan que este tratamiento es frecuentemente abandonado por los pacientes. Sin embargo, los recientes avances en el conocimiento de la fisiopatología de la disfunción vestibular han introducido nuevos métodos de RV (Bouccara, Sémont y Sterkers, 2003 y Norré y Beckers, 1988).

El proceso de adaptación durante la RV se caracteriza porque a pesar de mantener la excitación del receptor, desaparece la descarga aferente por la fibra sensorial primaria que ocasiona el vértigo. La habituación se relaciona con la pérdida de percepción de una sensación sin que desaparezca la descarga por la fibra primaria. La RV se fundamentará en un proceso de habituación, y su principio se basa en la realización de una serie de ejercicios o movimientos cuya finalidad es desencadenar el cuadro de vértigo que afecta al paciente, hasta que a través de la habituación se consiga la adaptación. Mediante la repetición frecuente de la circunstancia desencadenante, se logra la habituación o reducción de la respuesta a la sensación vertiginosa, hasta que finalmente ésta no ocurre (Bartual y colaboradores, 1999).

Una estrategia altamente efectiva es la liberación otolítica pero presenta un término de medio a alto en la recurrencia. Toledo, Cortés, Pane y Trujillo (2000), realizaron un estudio donde compararon la maniobra Semont, RV y la combinación de ambos. Este reveló que la maniobra Semont fue efectiva en el $80 \%$ de los pacientes que la recibieron a los quince días, versus el $45 \%$ de aquellos que solo recibieron RV. El tercer grupo que recibió la combinación de tratamientos, mostró una cura en el $100 \%$ de los casos cuando fueron evaluados a los tres meses, mientras que solo un $66 \%$ del grupo que recibió la maniobra Semont, fue encontrado asintomático $(p<0,05)$. Ellos concluyeron que esta es efectiva a corto plazo, pero la recurrencia es alta, mientras que la RV es efectiva y persistente a largo plazo, además la combinación de ambos tratamientos conduce a la remisión de los síntomas en un $100 \%$ tres meses posteriores al estudio. Por otro lado, Helminski y colaboradores (2005), realizaron un estudio con una duración de dos años, donde usaron ejercicios Brandt-Daroff para reducir la incidencia de reaparición del VPPB, pero este estudio encontró que una rutina diaria de ejercicios de Brandt-Daroff no afecta significativamente ( $>0.05)$, el tiempo de aparición (recurrencia) o la tasa de recurrencia de BBPV.

Norré y Beckers (1987), compararon dos grupos, uno que realizó método progresivo de rehabilitación vestibular (RV) y otro con un método de ejercicios fáciles (MF) que solo puede ser utilizado en BPPV. Los resultados después de una semana mostraron que el $52 \%$ de los pacientes del grupo MF no presentaron vértigo, mientras solamente el $32 \%$ del grupo con RV no lo presentaron, aunque estas diferencias no fueron significativas estadísticamente $(\mathrm{p}>0.05)$.

En cuanto a estudios donde se observe el comportamiento de otras variables, Evkall Hansson, Månsson, Ringsberg y Håkansson (2006), realizaron uno con veintinueve sujetos, con un grupo experimental y uno de control; donde midieron la discapacidad por el vértigo y equilibrio, y luego de tres meses de intervención con RV, el grupo experimental obtuvo mejorías significativas en diferentes situaciones como de pie sobre una pierna con los ojos abiertos $(\mathrm{p}=0.000)$, prueba de tándem $(\mathrm{p}=0.033)$ y discapacidad por vértigo $(\mathrm{p}=0.04)$. Asimismo, Mruzek, Barin, Nichols, Burnett y Welling (1995), estudiaron los efectos de la RV y el reforzamiento social (RS) sobre el equilibrio, valoración de la simetría del índice en una prueba de rotación, el cociente de la sensibilidad del movimiento (MSQ) y la discapacidad por vértigo en un grupo de sujetos con cirugía vestibular ablativa (laberintectomía vestibular), en tres subgrupos, uno recibió RV con RS, otro RV sin RS y el último grupo SR con ejercicios de movimiento articular. No hubo diferencias significativas en las distintas variables de los grupos después de ocho semanas de tratamiento, pero se reporta que los 
sujetos que recibieron RV con RS o si él, tuvieron menos sensibilidad al movimiento y mareos que el grupo que recibió ejercicios de movilidad articular.

Entonces de acuerdo a la evidencia científica los estudios con RV y ejercicios físicos no han demostrado el efecto de estos, sobre la discapacidad por vértigo con un programa semipresencial a corto plazo (cuatro semanas); por ello el propósito de este estudio se basa en determinar el efecto en el índice de discapacidad en pacientes adultos con VPPB mediante la RV con movimiento humano.

\section{METODOLOGÍA}

Sujetos: Participaron seis sujetos, de los cuales cuatro eran mujeres y dos hombres, con un promedio de edad de $49.5 \pm 14.22$ años, que fueron diagnosticados previamente con vértigo periférico, específicamente con VPPB, por un especialista en otorrinolaringología.

Instrumentos y materiales: En 1990, Jacobson desarrolló el Dizzness Handicap Inventory (DHI) o cuestionario de discapacidad por vértigo, el cual se propuso como una herramienta para cuantificar el impacto de este en las actividades y situaciones de la vida diaria. Es un instrumento que es ampliamente difundido y usado en todo el mundo, mide como estos síntomas afectan la calidad de vida del individuo. Además se encuentra estandarizado y se correlaciona confiablemente. En un estudio de Jacobson y colaboradores reportan una confiabilidad alta para pacientes vertiginosos $(\mathrm{r}=$ 0.97) (Ceballos y Vargas, 2004 y Hassid, Hennaux y Van Nechel, 2004).

El cuestionario consta de veinticinco preguntas, de las cuales nueve valoran aspectos emocionales (treinta y seis puntos), otras nueve valoran aspectos funcionales (treinta y seis puntos) y siete valoran aspectos físicos (veintiocho puntos), para obtener al final un puntaje máximo de cien puntos. Cada pregunta permite elegir entre tres respuestas: sí (cuatro puntos), algunas veces (dos puntos) y no (cero puntos). La discapacidad física se define como el grupo de desventajas que son experimentadas por un individuo como resultado del deterioro funcional y/u orgánico en uno $o$ varios sistemas. La funcional se manifiesta como la incapacidad de realizar tareas básicas que son asociadas con la vida diaria, en el campo profesional, ocupacional y recreativo. La emocional comprende las consecuencias psicológicas y/o psiquiátricas que surgen como resultado de patologías orgánicas, como por ejemplo, ansiedad, depresión o crisis de pánico, que alteran el desarrollo de las actividades cotidianas (Bouccara, Sémont y Sterkers, 2003).

La clasificación que se otorga para los aspectos emocionales y funcionales es: "sin discapacidad" de 0 a 14 puntos, "discapacidad moderada" de 15 a 24 puntos y "severa" de 25 puntos en adelante. En el aspecto físico se asignó una clasificación de "sin discapacidad" de 0 a 9 puntos, "discapacidad moderada" de 10 a 16 y de 17 en adelante "discapacidad severa". Por último el cuestionario se aplicó antes y después de terapia.

Procedimiento: Se contactaron seis sujetos que fueron diagnosticados con VPPB por un médico especialista en otorrinolaringología, los cuales persistieron sintomáticos a pesar del tratamiento médico. Se les otorgó un consentimiento el cual se les informó de forma escrita donde se les explicó en que consistía la RV.

La terapia vestibular duró cuatro semanas. Consistió en un mínimo de cuatro sesiones semanales de las cuales una de ellas era supervisada por un especialista. Durante la primera sesión se les hizo un pretest y se les explicó la ejecución correcta de los ejercicios de habituación, los de equilibrio y de acondicionamiento general que fueron contemplados en la terapia vestibular. Durante la última sesión se realizó el postest. 
Se realizaron movimientos para estabilizar la mirada, que fueron contempladas en los ejercicios de habituación y que además fomentan la capacidad adaptativa del sistema vestibular. También se realizaron ejercicios de control postural para obtener una correcta integración entre el sistema visual, vestibular y propioceptivo para el mantenimiento del equilibrio y la orientación en el espacio. De igual manera se ejecutaron actividades de acondicionamiento del estado general, las cuales consisten en procurar una mejoría en la autonomía del paciente para progresar en los aspectos funcionales y los físicos.

Análisis estadístico: Se aplicó estadística descriptiva para las variables en estudio, además se utilizó una t-student de medidas repetidas para comparar si existían diferencias estadísticamente significativas entre las dimensiones física, funcional y emocional entre el pre y postest, los datos fueron analizados con el SPSS versión 8.0 para Windows.

\section{RESULTADOS}

Se analizaron los resultados de seis sujetos, los cuales se sometieron a la terapia de RV durante un periodo de cuatro semanas. Se observó que antes de iniciar la terapia dos sujetos se encontraban con discapacidad física moderada, mientras que los restantes cuatro presentaban física severa. Posterior a esta los dos que presentaban discapacidad moderada, se presentaron sin ella, y de los cuatro que presentaban severa, dos presentaron moderada y los otros dos continuaron con severa pero con un menor puntaje.

En cuanto al aspecto funcional, cuatro sujetos no presentaron discapacidad, uno presentó moderada y el otro severa. Luego de someterse a la rehabilitación, el sujeto que presentaba discapacidad funcional moderada no la presentó y el que la presentaba severa presentó moderada. Los restantes cuatro sujetos continuaron sin discapacidad funcional.

En el aspecto emocional, únicamente un sujeto presentó discapacidad moderada, mientras que los restantes cinco sujetos no presentaron discapacidad. El sujeto con moderada en el pretest no la presentó en el postest.

Se realizó una $t$-student de medidas repetidas para las distintas dimensiones que fueron valoradas. En cuanto a la dimensión física, hubo diferencias estadísticamente significativas entre el pretest $(19.33 \pm 4.67$ puntos $)$ y el postest $(13 \pm 7.24$ puntos $)(t=2.65 ; \mathrm{p}<0.05)$, es decir los sujetos disminuyeron el deterioro físico-funcional después de la aplicación del tratamiento. No hubo diferencias significativas en las dimensiones funcional ( $t: 2.44 ; p>0.05)$, emocional ( $t: 2.37 ; p>0.05)$ y general (t: $2.55 ; \mathrm{p}>0.05)$. 
Gráfico 1. Diferencias en la dimensión física de los pacientes con vértigo entre el pretest y el postest

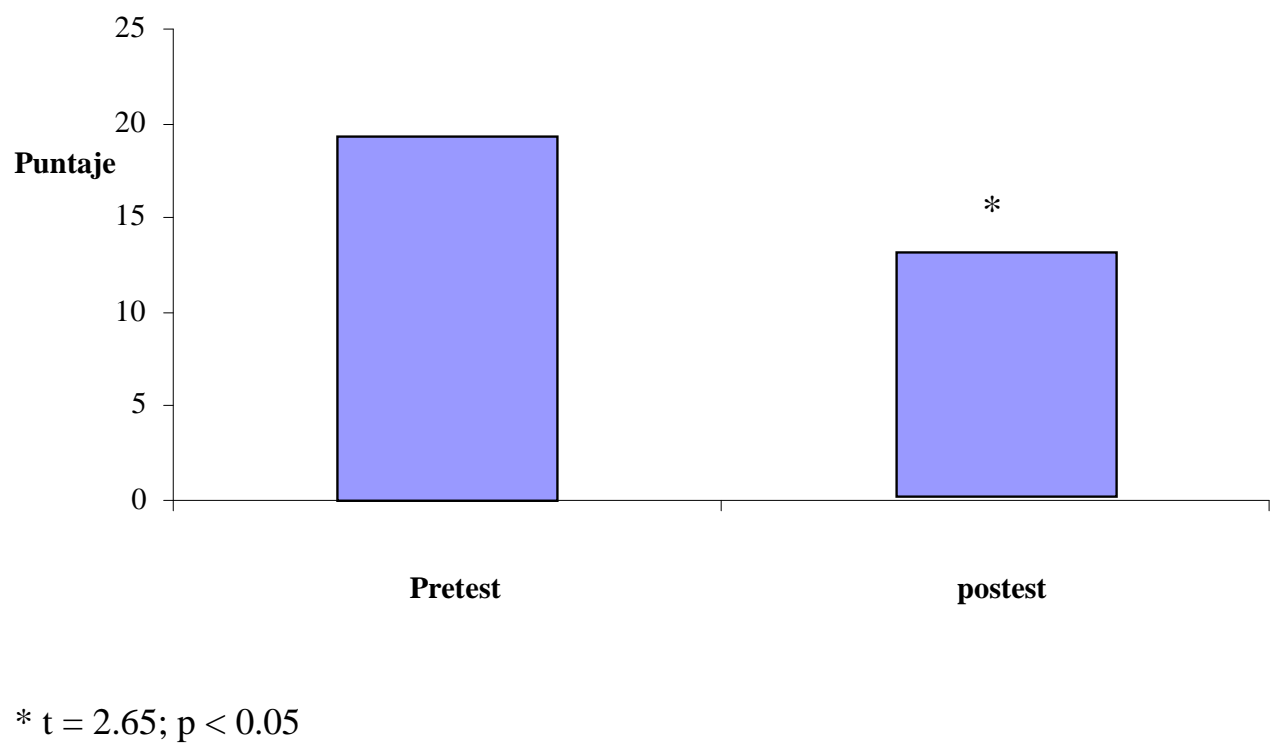

\section{DISCUSIÓN}

El propósito de este estudio se basa en determinar el efecto en el índice de discapacidad en pacientes adultos con vértigo paroxístico posicional benigno, mediante la RV con el movimiento humano.

Este estudio encontró que luego del tratamiento hubo una mejoría significativa, estadísticamente, en el índice de discapacidad por vértigo en la dimensión física entre el pretest y el postest, es decir, los sujetos disminuyeron el deterioro físico-funcional después de la aplicación del tratamiento. En cuanto a las dimensiones funcional, emocional y en las suma de las tres se muestra que aunque no hubo diferencias significativas. Dicho fenómeno pudo suceder debido a que la muestra fue muy pequeña $(\mathrm{n}=6)$, donde únicamente un sujeto presentaba discapacidad emocional, cuatro funcional y todos física.

Esta mejoría en la dimensión física puede deberse a que la compensación vestibular, como proceso, supone una reorganización anatómica y funcional de este sistema de las áreas cerebrales conectadas a él. No es una restauración perfecta, completa del comportamiento motor y de las capacidades perceptivas previas a la lesión pero permite aparentemente una tendencia a mejorar dichas facultades. Se relaciona con la habilidad que tiene el sistema nervioso central para procesar información proveniente de un sistema sensorial deficitario y responder adecuadamente a los requerimientos que continuamente genera el mantenimiento del equilibrio (Bartual y colaboradores, 1999; Bouccara, Sémont, Sterkers, 2003; Hassid, Hennaux y Van Nechel, 2004 y Mruzek y colaboradores, 1995). Lo anterior es apoyado por Santandreu (2004) y López (2004) que afirman que existen estudios que han demostrado la efectividad de la RV en pacientes con VPPB y contrario a Helminski y colaboradores (2005) y Norré y Beckers (1987), quienes en sus estudios no encontraron resultados significativos al utilizar la RV. Asimismo, se encontró que con solo cuatro semanas de tratamiento se puede dar resultados significativos, tiempo relativamente corto, y es un resultado que no fue encontrado en el estudio de Toledo, Cortés, Pane y Trujillo (2000), donde 
encontraron que la maniobra Semont era efectiva a corto plazo, pero que la RV era efectiva y persistente solamente a largo plazo. Específicamente al resultado que se ha encontrado en la dimensión física Evkall Hansson y colaboradores (2006) si se hallaron resultados significativos a tres meses plazo, mientras que Mruzek y colaboradores (1995) después de ocho semanas de tratamiento no encontraron mejorías significativas.

\section{CONCLUSIONES}

La RV mejoró el índice de discapacidad en la dimensión física en sujetos con VP luego de cuatro semanas de tratamiento.

No se encontraron mejorías en la dimensión emocional de los sujetos, solo tendencias a ello.

La RV bajo una modalidad semipresencial a corto plazo, o sea constituye una herramienta efectiva y práctica para el tratamiento de síndromes vertiginosos.

\section{REFERENCIAS}

Bartual, J., Pérez, N., Baharona de Guzman, R., Fernández, F., Guirado, F., Manrique, M., Oliva, M., Roquete, J., Sánchez, J., Suárez, C., (1998). El sistema vestibular y sus alteraciones Tomo 1. Barcelona: Masson.

Bartual, J., Pérez, N., Baharona de Guzman, R., Garcia-Ibañez, E., Gelabert, M., Irinia, P., Algarra, J., Pérez, B., Ramírez, R., Roquette, J., Sánchez, J., Santos, S., Saenz, R., Soto, A., Urrestarazú, E., Viteri, C. (1999). El sistema vestibular y sus alteraciones Tomo 2. Barcelona: Masson.

Bouccara, D., Sémont, A. y Sterkers, O. (2003). Rééducation Vestibulaire. (Encyclopédie MédicoChirurgicale Oto-Rhinolaryngologie 20-206-A-10-2003, 7p.) Paris: Edtions Scientifiques et Médicales Elsevier SAS.

Ceballos, R. y Vargas A. (2004). Aplicación y utilidad del Dizziness Handicap Inventory en pacientes con vértigo del Servicio de Otorrinolaringología del Hospital de Especialidades del Centro Médico Nacional Siglo XXI. Fecha de acceso: 22 de marzo del 2007. Fuente: www.medigraphic.com/pdfs/abc/bc-2004/bc044d.pdf.

Evkall Hansson, E., Månsson, N.O., Ringsberg, K. A. y Håkansson, A. (2006). Dizziness among patients with whiplash-associated disorder: a randomized controlled trial. J Rehabil Med. 38(6):387-90.

Hassid, N., Hennaux, C. y Van Nechel, C. (2004). La rééducation vestibulaire. Paris: Éditions Frison-Roche.

Hassid, N. (2002). Rehabilitation of the vertigo patient. Rev Med Brux. Sep, 23(4): A36871.

Helminski, J.O., Janssen, I., Kotaspouikis, D., Kovacs, K., Sheldon, P., McQueen, K. y Hain, T.C. (2005). Strategies to prevent recurrence of benign paroxysmal positional vertigo. Arch Otolaryngol Head Neck Surg. Apr; 131(4):344-8. 
López, A. (2004). El mareo mejora con rehabilitación. Fecha de acceso: 8 de junio del 2007. Fuente:

www.elmundosalud.elmundo.es/elmundosalud/2004/10/25/medicina/1098690385.html.

Norré, M.E. y Beckers, A.(1987). Exercise treatment for paroxysmal positional vertigo: comparison of two types of exercises. Arch Otorhinolaryngol. 244(5): 291-4.

Norré, M.E. y Beckers, A. (1988). Benign paroxysmal positional vertigo in the elderly. Treatment by habituation exercises. J Am Geriatr Soc. May;36(5):425-9.

Mruzek, M., Barin, K., Nichols, D.S., Burnett, C.N. y Welling, D.B.(1995). Effects of vestibular rehabilitation and social reinforcement on recovery following ablative vestibular surgery. Laryngoscope. 105(7 Pt 1), 686-92.

Oghalai, J.S., Manolidis, S., Barth, JL., Stewart, M.G., y Jenkins, H.A. (2000). Unrecognized benign paroxysmal positional vertigo in elderly patients. Otolaryngol Head Neck Surg. 122:630-634.

Roman, S., Thomassin, JM. (2000). Physiologie Vestibulaire. (Encyclopédie Médico-Chirurgicale Oto-Rhinolaryngologie 20-198-A-10,2000, 14p.) Paris: Edtions Scientifiques et Médicales Elsevier SAS.

Santandreu, M. (2004). Tratamiento rehabilitador de los trastornos del equilibrio de origen vestibular. Fecha de acceso: 8 de junio del 2007. Fuente: www.svmfr.com/reuniones/XVI/trabajos/ponencias/ TRATAMIENTO\%20REHABILITADOR\%20DE\%20LOS\%20TRASTORNOS\%20DEL \%20EQUILIBRIO\%20DE\%20ORIGEN\%20VESTIBULAR.pdf.

Toledo, H., Cortés, M.L., Pane, C. y Trujillo, V. (2000). Semont maneuver and vestibular rehabilitation exercises in the treatment of benign paroxysmal postural vertigo. A comparative study. Neurología., Apr; 15(4):152-7.

Toupet, M., Imbaud-Genieys S. (2002). Examen clinique et paraclinique du patient atteint de vertiges et/ou de troubles de l'équilibre. (Encyclopédie Médico-Chirurgicale OtoRhinolaryngologie 20-199-A-10,2002, 21p.) Paris: Edtions Scientifiques et Médicales Elsevier SAS.

Tran Ba Huy, P., de Waele, C. (1996). Les vertiges et le praticien. Paris : Éditions John Libbey Eurotext.

Whitney, S.L., Hudak, M. y Marchetti, G. (1999). The activities-specific balance confidence scale and the dizziness handicap inventory: a comparison. Journal of Vestibular Research. 9(4), $253-259$.

Fecha de recepción del artículo: 07 de mayo del 2007.

Fecha de aceptación del artículo: 10 de julio del 2007.

Fecha de publicación del artículo: 31 de julio del 2007. 